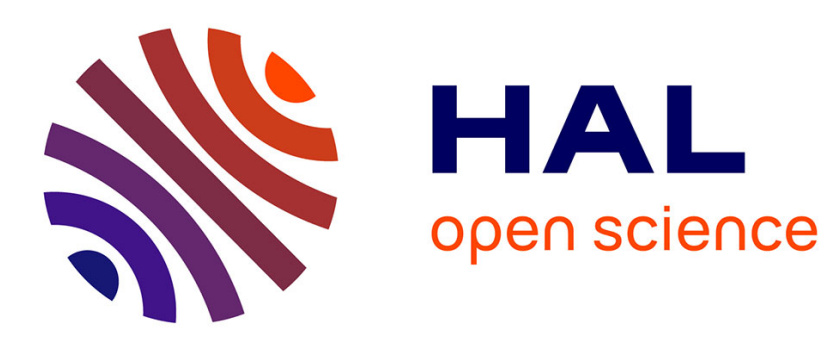

\title{
Influence of Intrapredatory Interferences on Impulsive Biological Control Efficiency
}

Sapna Nundloll, Ludovic Mailleret, Frédéric Grognard

\section{To cite this version:}

Sapna Nundloll, Ludovic Mailleret, Frédéric Grognard. Influence of Intrapredatory Interferences on Impulsive Biological Control Efficiency. Bulletin of Mathematical Biology, 2010, 72 (8), pp.2113-2138. 10.1007/s11538-010-9531-6 . hal-00847302

\section{HAL Id: hal-00847302 https://hal.inria.fr/hal-00847302}

Submitted on 23 Jul 2013

HAL is a multi-disciplinary open access archive for the deposit and dissemination of scientific research documents, whether they are published or not. The documents may come from teaching and research institutions in France or abroad, or from public or private research centers.
L'archive ouverte pluridisciplinaire $\mathbf{H A L}$, est destinée au dépôt et à la diffusion de documents scientifiques de niveau recherche, publiés ou non, émanant des établissements d'enseignement et de recherche français ou étrangers, des laboratoires publics ou privés. 


\title{
Influence of intrapredatory interferences on impulsive biological control efficiency
}

\author{
Sapna Nundloll ${ }^{\mathrm{a}}$, Ludovic Mailleret ${ }^{\mathrm{b}}$, Frédéric Grognard $^{\mathrm{a}}$ \\ ${ }^{a}$ INRIA, COMORE Team, Sophia Antipolis, France \\ ${ }^{b}$ INRA, UR 880, Sophia Antipolis, France
}

\begin{abstract}
In this paper, a model is proposed for the biological control of a pest by its natural predator. It incorporates a qualitative description of intrapredatory interference whereby predator density decreases the per capita predator efficiency and generalises the classical Beddington-DeAngelis formulation. A pair of coupled ordinary differential equations are used, augmented by a discrete component to depict the periodic release of a fixed number of predators into the system. This number is defined in terms of the rate of predator release and the frequency at which the releases are to be carried out. This formulation allows us to compare different biological control strategies in terms of release size and frequency that involve the same overall number of predators. The stability properties of the zero-pest solution are analysed. We obtain a biological condition on the interference strength and a minimal bound on the predator release rate required to eradicate a pest population. We demonstrate that increasing the frequency of releases reduces this minimal rate and also increases the rate of convergence of the system to the zero-pest solution for a given release rate. Additionally, we show that other conclusions are to be expected if the interferences between predators have weaker or stronger effects than the generalised Beddington-DeAngelis formulation proposed in this paper.

Key words: Impulsive differential equations, nonlinear systems, predator-prey dynamics, Beddington-DeAngelis, sector conditions
\end{abstract}

\section{Introduction}

Biologically controlling pest invertebrates using their natural enemies possesses numerous advantages over control methods that involve the use of chemicals $[6,33]$. The aim of mathematically modelling the population dynamics is to assist in the identification of more effective testing protocols of biological control measures to inform field implementation. This paper presents a general model of an inundative biological control program, which consists of regular releases of natural enemies to eliminate the pest organisms. In particular, it analyses the disruptive effects of intraspecific interference within the enemy population on the pest management program.

Email addresses: sapna.nundloll@inria.fr (Sapna Nundloll), ludovic.mailleret@sophia.inra.fr (Ludovic Mailleret), frederic.grognard@inria.fr (Frédéric Grognard) 
Intraspecific interference, or competition, is well-documented in the biological control literature. Several species of arthropods, used as natural enemies, display territorial behaviour that disrupts the food searching ability of their conspecifics and thus impacts the functional response [18, 40, 7, 13]. Insects of the Hymenoptera and Coleoptera order, for instance, emit oviposition deterring volatile pheromones at high population densities - a process which has a high energetic cost and as a consequence of which, the organism is believed to be less efficient in prey searching and handling $[41,7]$. Passive substrate marking, where a given individual is detered from foraging a given territory marked by conspecifics feces, has also been reported in [1]. Interfering behaviours are also observed among predatory mites (order Acari) that are widely used against various pests in orchards and greenhouse crops [12, 14]. Whilst at normal levels in the field, interference effects are not as apparent as in laboratory conditions [29, 38], their potential impact in a given biological control program - due to the sheer numbers or density of biological control agents involved - cannot be ignored (see for instance [8]).

Such interference effects modify the trophic response and are therefore modelled by incorporating a penalising term related to the predator population size. In [15] and [34], the influence of this type of competition was considered to be exponential with respect to the predator density. Other models by Beddington [3] and DeAngelis [9], as well as more recent ones such as [35], involved altering the Holling type II response [16]. Arditi et al. [2] proposed the ratio-dependent trophic function, which also displayed a similar interference property. To compare ratio- and densitydependence, Buffoni et al. presented a generalised version of these models by qualitatively defining the trophic functions [5, 4].

To generalise further the density-dependent formulation in $[5,4]$, we propose, in this paper, to use sector conditions, common in the control engineering and neural networks literature: these conditions bound nonlinearities between two linear functions and the obtained stability results, termed "absolute stability", show that the considered system is stable for any nonlinearity that satisfies the sector condition (see [25] for the founding paper, [23] for a review, [39, 19] for textbook presentations and [10] for current research on the topic). This phenomenological description aims to minimise the risks of modelling artefactslinked to the use of specific forms for the modelling of the interference, whilst giving enough information on the general properties of the system. It provides sufficient detail for realistic interpretations of the results to be possible and gives guidelines for the selection and deployment of biological control agents.

Furthermore, our modelling approach accounts for the inherently hybrid dynamics involved: the intrinsic predatorprey dynamics are described using a pair of ordinary differential equations, which are augmented by a discrete component depicting the predator releases. We seek to answer two questions: firstly, what conditions are required for the pest population to be eliminated and secondly, what release strategy is to be employed for this eradication be as quick as possible. In our work, the size of a release is defined explicitly as the product of the rate of predator release per unit time and the release period (see also [26, 31]). By doing so, we are able to compare different biological control strategies in terms of the release size and release frequency that involve the same overall number of predators.

Merging the continuous and discrete dynamics together yields a model that is said to be impulsive (or semi- 
discrete) and that is used in various fields of mathematical biology for instance in vaccination modelling [36], cancer treatment modelling [22], or in the description of biological mechanisms that yield classical discrete time population models [11]. We refer to [28] for a review of the topic. In agricultural pest control, the impulsive changes can be due to the release of natural enemies [26], as well as harvesting [31] or pesticide usage [24, 37]. Other studies, involving semi-discrete predator-prey dynamics, that also take into account intrapredatory competition of the type presented here (that is, involving a modification of the trophic response) consider explicit Beddington-DeAngelis responses $[30,43,32,42]$. As will be shown subsequently, the qualitative formulation in this paper is able to provide us with general insights on the influence of intrapredatory interference on an impulsive system.

This paper is structured as follows. Section 2 introduces our model. In Section 3, we demonstrate the existence of a periodic pest-free solution driven by the predator releases and compute the conditions required for its stability: one is related to the biology of the predator species while the other defines a minimal predator release rate that guarantees pest eradication. In Section 4, the position of our result with respect to other kinds of interference models is discussed. In particular, we briefly show that the results we obtain through the sector conditions do not hold if intrapredatory interference is weaker or stronger. In Section 5.1, coming back to our modelling framework, the minimal release rate is shown to be an increasing function of the release period. it is shown subsequently in Section 5.2 that the speed of eradication decreases with the release period. In each section, the results are presented first for a simpler version than the one allowed by the general formulation. These are then extended, using sector conditions, to a more general qualitative case. Finally in Section 6, we make some concluding remarks on the results of this work.

\section{The model}

The predator-prey dynamics are modelled by two ordinary differential equations that are augmented with an impulsive component which depicts the periodic predator releases. In the following, $x$ and $y$ are the pest and predator populations respectively,

$$
\left\{\begin{array}{l}
\dot{x}=f(x)-g\left(\frac{\alpha(x)}{\beta(y)}\right) y \\
\dot{y}=h\left(\frac{\alpha(x)}{\beta(y)}\right) y-m y \\
y\left(n T^{+}\right)=y(n T)+\mu T, \quad \text { where } n \in \mathbb{N},
\end{array}\right.
$$

with non-negative initial conditions $x_{0}$ and $y_{0}$. The + -superscript indicates the instant right after a predator release of intensity $\mu T$; the $f(),. g($.$) and h($.$) functions are the pest-growth, functional and numerical responses respectively;$ $m$ is a positive constant which represents the natural mortality rate of the predators.

The functions $f(x), g\left(\frac{\alpha(x)}{\beta(y)}\right) y$ and $h\left(\frac{\alpha(x)}{\beta(y)}\right) y$ are all assumed locally Lipschitz continuous for $x, y \geq 0$ so that the continuous-time part of the model has unique solutions. They are qualitatively defined according to Hypotheses 1 below. The prime indicates their derivative with respect to their argument.

\section{Hypotheses 1.}


a. $f(0)=0, f^{\prime}(0)>0$;

$\exists \bar{x}$ such that $\forall x \geq \bar{x}, f(x)<0$;

$f(x) / x$ is upper bounded for $x \geq 0$, such that $S=\sup _{x \geq 0}(f(x) / x)$;

b. $g(0)=0, g^{\prime}(0)>0$ and $\forall s>0, g(s)>0$;

c. $h(0)=0$ and $\forall s>0, h(s) \geq 0$.

$f(x)$ is the pest growth rate. We consider that there is no spontaneous generation of the pest, that it does not suffer from strong Allee effect (i.e. negative growth at low densities) and that its population growth stops at $\bar{x}$. In case it overshoots, the environmental conditions naturally bring it back under $\bar{x}$ what is typical of logistic-like growth functions. The per capita growth rate $f(x) / x$ is also considered to be finite at all population sizes, which is a classical hypothesis.

$g($.$) and h($.$) are the functional and numerical responses respectively which represent the elimination of pests$ due to predation and the resulting increase in the predator population. We refer to both as the trophic functions as they are involved in the transfer of biomass from the pest to the predator population. Actually, a large part of population dynamics models assume proportionality between functional and numerical responses. There are however few biological reasons to impose this strict relationship, although both functions should present the same qualitative properties. The trophic functions vary according to both the pest and the predator population sizes. This dependence is parameterised by the mappings $\alpha(x)$ and $\beta(y)$, which capture the sensitivity of the functions $g($.$) and h($.$) to the$ two populations. These two parameter functions $\alpha(x)$ and $\beta(y)$ are assumed to verify affine sector conditions (as in $[39,17])$ such that for all $x, y \geq 0$

$$
\begin{aligned}
a_{1} x & \leq \alpha(x) \leq a_{2} x, \\
b_{1} y+b_{0} & \leq \beta(y) \leq b_{2} y+b_{0},
\end{aligned}
$$

where $a_{1}, a_{2}, b_{1}, b_{2}>0$ and $b_{0} \geq 0$. Parameters $a_{i}$ describe the sensitivity of the trophic functions to the pest density: a higher $a_{i}$ indicates better capacities of the predators in consuming the pests and growing on them. For $i>0$, the $b_{i}$ describe the strength of intraspecific interference among predators: the higher the $b_{i}$, the stronger the interference between predators. $b_{0}$ allows us to discriminate between density-dependent and ratio-dependent interferences.

The Hypotheses $1 \mathrm{~b}$ and $1 \mathrm{c}$ on $g($.$) and h($.$) together with (2) imply that there is neither predation nor predator$ growth in the absence of pests. Predation becomes immediately positive upon a pest invasion, then remains nonnegative for all positive pest population. The numerical response of the predators is on its part only assumed to be non-negative in the presence of pests. The trophic functions argument follows a generally increasing trend with respect to $x$ as given by $\alpha(x)$. Conversely, increasing predator numbers penalises this argument according to the strength of intraspecific competition given by $\beta(y)$, so that it generally tends to decrease with $y$. As recalled in the introduction, the latter property is typical of intrapredatory interference.

Figure 1 shows three examples of nonlinear $\alpha(x)$ and $\beta(y)$ functions bounded within a sector defined as in condition (2). The Lipschitz square-like fluctuations depicted for both functions provide a rather extreme example for the 

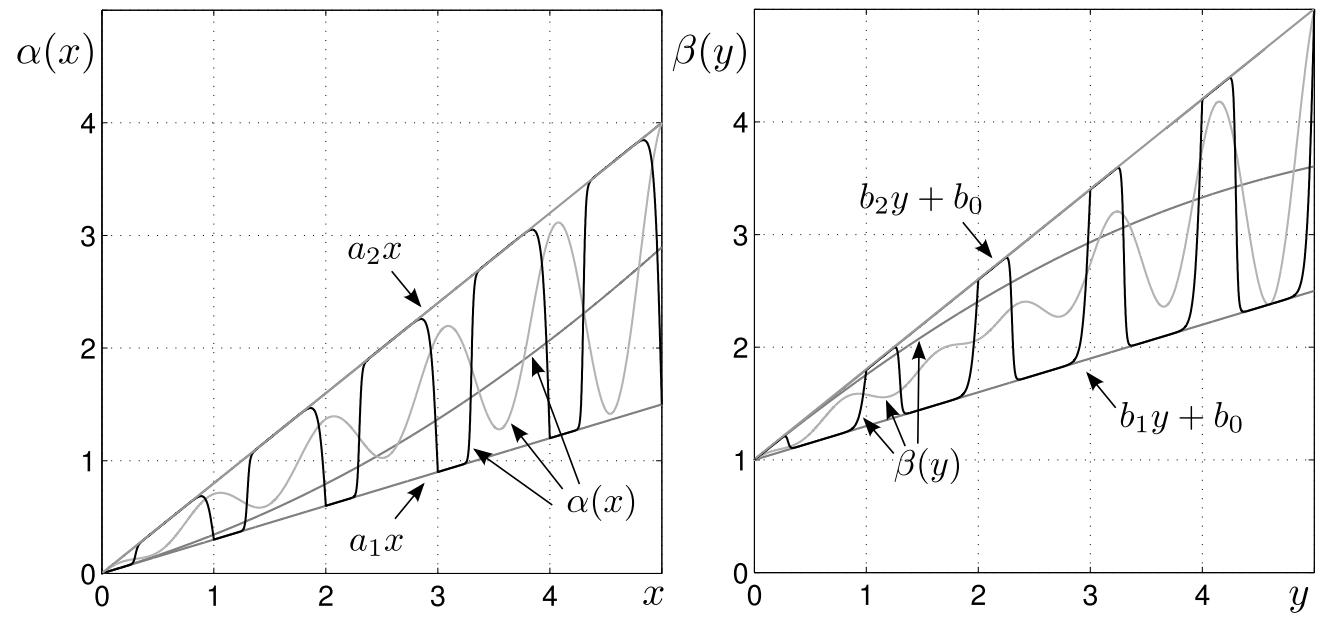

Figure 1: Three examples of $\alpha(x)$ (left) and $\beta(y)$ (right) verifying sector conditions (2)

purpose of our analysis and are not expected to occur in nature.

Remark: We note here that if the trophic responses are Type II Holling curves [16] (that clearly verify hypothesis $H 1 b$ and H1c) and if $\alpha(x)$ and $\beta(y)$ are affine, that is $a_{1}=a_{2}=a, b_{1}=b_{2}=b$ in (2), one recovers a classical Beddington-DeAngelis expression. Indeed, Holling II curves are of the form

$$
g(s)=\frac{\zeta s}{1+\theta s}
$$

with $\zeta$ and $\theta$ some (positive) parameters. Then,

$$
g\left(\frac{\alpha(x)}{\beta(y)}\right)=\frac{\zeta \alpha(x)}{\beta(y)+\theta \alpha(x)},
$$

which gives with affine $\alpha(x)$ and $\beta(y)$,

$$
g\left(\frac{\alpha(x)}{\beta(y)}\right)=\frac{\zeta a x}{b_{0}+b y+\theta a x}=\frac{\left(\frac{\zeta a}{b_{0}}\right) x}{1+\left(\frac{b}{b_{0}}\right) y+\left(\frac{\theta a}{b_{0}}\right) x}
$$

which is the Beddington-DeAngelis trophic response's analytical form [3, 9].

Notice moreover that if $b_{0}=0$, one obtains ratio-dependent (or Arditi-Ginzburg) trophic responses [2]. The formulation proposed in the present paper is inspired by and provides a generalised version of the one presented in [4].

The impulsive component (predator releases) is described by the third equation in (1). The number of predators injected at these instants is defined as the product of the predator release rate $\mu$ and the release period T. Notice that strictly speaking, $\mu$ is rather a mean release rate i.e. the mean number of predator released per unit time over a long time period; we shall however stick to the denomination "release rate" for $\mu$ in the following. Predator releases 
modelled thus, we have decoupled the two characteristics of the biological control strategy to implement namely: the number of predators we are to invest in per unit time and, for a given rate, how to distribute this number over time. This allows us to compare the effect of few but large releases with more frequent ones of smaller size.

Finally we note that with Hypotheses 1 and conditions (2), (1) is a positive system, that is its variables stay in the non-negative orthant if initiated therein.

\section{Stability of the zero-pest solution}

In this section, we demonstrate the existence and stability of a pest-free solution of system (1). This solution is of interest to us because it is the target state in our biological control program.

We first define this solution in Proposition 1 below.

Proposition 1. Let Hypotheses 1 and conditions (2) be satisfied. Then,

$$
\left.\left(x_{p}(t), y_{p}(t)\right)=\left(0, y^{*} e^{-m(t} \bmod T\right)\right),
$$

where

$$
y^{*}=\frac{\mu T}{1-e^{-m T}},
$$

is a periodic solution of model (1).

Proof: When considering zero-pest solutions, model (1) becomes

$$
\left\{\begin{array}{l}
\dot{x}=0, \\
\dot{y}=-m y, \\
\forall n \in \mathbb{N}, y\left(n T^{+}\right)=y(n T)+\mu T,
\end{array}\right.
$$

that yields: $y\left((n+1) T^{+}\right)=y\left(n T^{+}\right) e^{-m T}+\mu T$. This sequence $\left(y\left(n T^{+}\right)\right)_{n \in \mathbb{N}}$ has a single equilibrium $y^{\star}=\mu T /(1-$ $\left.e^{-m T}\right)$, which concludes the proof.

In order to identify the conditions for the stability of solution (3a), we state two preliminary propositions on pest growth (Proposition 2) and on the functional response (Proposition 3).

Proposition 2. Let Hypotheses 1 and conditions (2) be satisfied. Then there exists $t_{f}\left(x_{0}, y_{0}\right) \geq 0$ such that $\frac{\alpha(x(t))}{\beta(y(t))} \in$ $\left[0, \frac{2 a_{2} \bar{x}}{2 b_{0}+b_{1} y^{*} e^{-m T}}\right]$ for $t \geq t_{f}\left(x_{0}, y_{0}\right)$.

Proof: Notice that from (2): $\frac{\alpha(x)}{\beta(y)} \leq \frac{a_{2} x}{b_{0}+b_{1} y}$. Now, we first seek an upper-bound for $x$ and then a lower-bound for $y$.

Firstly suppose $x_{0} \leq \bar{x}$. Since $f(\bar{x})<0, \dot{x}(x=\bar{x})<0$. Therefore, $\forall t \geq 0, x\left(t, x_{0}\right) \leq \bar{x}$. Next, suppose $x_{0}>\bar{x}$. $g() \geq$.0 for $x, y \geq 0$ and $f(x)<0$ for $x \geq \bar{x}, \dot{x}=f(x)-g(). y \leq \max _{\bar{x} \leq x \leq x_{0}} f(x)$. This is a negative constant, so that $x$ decreases into $x \in[0, \bar{x}]$ in finite time $t_{1}\left(x_{0}\right)$. 
Secondly, consider the new coordinate $\tilde{y}=y-y_{p}$ whose dynamics are

$$
\dot{\tilde{y}}=h\left(\frac{\alpha(x)}{\beta(y)}\right) y-m y+m y_{p}=h\left(\frac{\alpha(x)}{\beta(y)}\right) y-m \tilde{y} \geq-m \tilde{y},
$$

where the impulsive component vanishes as

$$
\tilde{y}\left(n T^{+}\right)=y(n T)+\mu T-y_{p}(n T)-\mu T=\tilde{y}(n T) .
$$

Since, by construction, $\tilde{y} \geq-y^{*}$, this differential inequality implies that there exists a finite time $t_{2}\left(y_{0}\right)$ such that $\tilde{y}(t) \geq-\frac{y^{*} e^{-m T}}{2}$. After this finite time, we then have that $y(t)=\tilde{y}(t)+y_{p}(t) \geq-\frac{y^{*} e^{-m T}}{2}+y^{*} e^{-m T}=\frac{y^{*} e^{-m T}}{2}$.

This implies that $\frac{\alpha(x)}{\beta(y)} \in\left[0, \frac{2 a_{2} \bar{x}}{2 b_{0}+b_{1} y^{*} e^{-m T}}\right]$ for $t \geq t_{f}\left(x_{0}, y_{0}\right)=\max \left(t_{1}\left(x_{0}\right), t_{2}\left(y_{0}\right)\right)$.

Proposition 3. Let $\sigma(s, q)=g^{\prime}(0) \frac{q s}{q+s}$ where $q>0$. Then for all $K>0$, there exists $q$ such that $\forall s \in[0, K], \sigma(s, q) \leq g(s)$, and $\sigma^{\prime}(0, q)=g^{\prime}(0)$.

Proof: The proof for the existence of $\sigma(s, q)$ is constructed in two parts on the interval $[0, K]$. We note that

$$
\sigma^{\prime}(0, q)=g^{\prime}(0)
$$

Let $q^{*}>0$ be such that

$$
\sigma^{\prime \prime}\left(0, q^{*}\right)=\frac{-2 g^{\prime}(0)}{q^{*}} \triangleq g^{\prime \prime}(0)
$$

Then

$$
\begin{aligned}
\sigma\left(s, q^{*}\right)-g(s) & =\sigma\left(0, q^{*}\right)+\sigma^{\prime}\left(0, q^{*}\right) s+\frac{\sigma^{\prime \prime}\left(0, q^{*}\right)}{2} s^{2}-g(0)-g^{\prime}(0) s-\frac{g^{\prime \prime}(0)}{2} s^{2}+O\left(s^{3}\right), \\
& =\frac{s^{2}}{2}\left(\sigma^{\prime \prime}\left(0, q^{*}\right)-g^{\prime \prime}(0)\right)+O\left(s^{3}\right) .
\end{aligned}
$$

Therefore for the chosen $q^{*}$ there exists $\varepsilon>0$ such that $\forall s \in[0, \varepsilon), \sigma\left(s, q^{*}\right)-g(s) \leq 0$. Furthermore, $\sigma(s, q)$ is an increasing function in $q$ so that $\forall s \in[0, \varepsilon), \forall q \in\left(0, q^{*}\right], \sigma(s, q) \leq g(s)$. Next, we consider the interval $s \in[\varepsilon, K]$. Here, since for any given $s, \lim _{q \rightarrow 0^{+}} \sigma(s, q)=0$, it suffices to choose a sufficiently small $q \leq q^{*}$ such that

$$
\max _{s \in[\varepsilon, K]} \sigma(s, q) \leq \min _{s \in[\varepsilon, K]} g(s)
$$

and thus

$$
\sigma(s, q) \leq g(s) \forall s \in[0, K]
$$

Our analysis of the stability of (3a) for affine $\alpha(x)$ and $\beta(y)$ is presented in detail in Section 3.1. The results are then extended to the case when these are nonlinear in Section 3.2. 


\subsection{Affine parameterisation}

Let $\alpha(x)=a x$ and $\beta(y)=b y+b_{0}$, where $a, b$ are positive constants. These functions thus defined, yield a generalisation of the Beddington-DeAngelis system (it would reduce to it if $g($.$) and h($.$) were of Holling II type). As such,$ we note that this formulation generalises the results by [43] and [32]. The stability conditions are then described by the following theorem.

Theorem 1. The solution $\left(0, y_{p}(t)\right)$ of (1) is LAS if and only if

$$
f^{\prime}(0)<\frac{a g^{\prime}(0)}{b},
$$

and

$$
\mu>\frac{b_{0}}{b}\left(\frac{1-e^{-m \frac{b f^{\prime}(0)}{a g^{\prime}(0)} T}}{e^{-m \frac{b f^{\prime}(0)}{a g^{\prime}(0)} T}-e^{-m T}}\right)\left(\frac{1-e^{-m T}}{T}\right) .
$$

Furthermore, with $q$ chosen as in Proposition 3 with $K=\frac{2 a_{2} \bar{x}}{2 b_{0}+b_{1} y^{*} e^{-m T}}$, the solution is GAS if

$$
S<\frac{a g^{\prime}(0)}{b},
$$

and

$$
\mu>\frac{q b_{0}+a \bar{x}}{q b}\left(\frac{1-e^{-m \frac{b S}{a g^{\prime}(0)} T}}{e^{-m \frac{b S}{a g^{\prime}(0)} T}-e^{-m T}}\right)\left(\frac{1-e^{-m T}}{T}\right) .
$$

Proof: The local stability conditions can be identified by applying Theorem 1 from [22]. After some computations, we obtain as necessary and sufficient conditions $e^{-m \int_{0}^{T} d \tau}<1$ and $e^{\int_{0}^{T}\left(f^{\prime}(0)-\frac{g^{\prime}(0) \alpha^{\prime}(0) y_{p}(\tau)}{\beta\left(y_{p}(\tau)\right)}\right) d \tau}<1$. The former is trivial while the latter yields

$$
\int_{0}^{T}\left(\frac{\alpha^{\prime}(0) y_{p}(\tau)}{\beta\left(y_{p}(\tau)\right)}\right) d \tau>\frac{f^{\prime}(0)}{g^{\prime}(0)} T .
$$

We note that in this equation $\alpha^{\prime}(0)=a$ and $\beta\left(y_{p}(\tau)\right)=b y^{*} e^{-m \tau}+b_{0}$, so

$$
\begin{gathered}
\int_{0}^{T}\left(\frac{a y^{*} e^{-m \tau}}{b y^{*} e^{-m \tau}+b_{0}}\right) d \tau>\frac{f^{\prime}(0)}{g^{\prime}(0)} T, \\
\Leftrightarrow \quad\left(e^{-m \frac{b f^{\prime}(0)}{a g^{\prime}(0)} T}-e^{-m T}\right) b y^{*}>b_{0}\left(1-e^{-m \frac{b f^{\prime}(0)}{a g^{\prime}(0)} T}\right) .
\end{gathered}
$$

Now, the right-hand side of the above inequality is non-negative, which requires that the left-hand side be positive. Thus for the stability condition to hold we need that

$$
e^{-m \frac{b f^{\prime}(0)}{a g^{\prime}(0)} T}-e^{-m T}>0,
$$

from which we obtain the necessary condition (5a). Using (3b) and substituting in (7), and finally rearranging, we obtain

$$
\mu>\frac{b_{0}}{b}\left(\frac{1-e^{-m \frac{b f^{\prime}(0)}{a g^{\prime}(0)} T}}{e^{-m \frac{b f^{\prime}(0)}{a g^{\prime}(0)} T}-e^{-m T}}\right)\left(\frac{1-e^{-m T}}{T}\right),
$$


which is condition (5b).

To establish the condition for global stability of (3a), we define a new system of coordinates $\tilde{x}=x-x_{p}$ and $\tilde{y}=y-y_{p}$ (this variable was already considered in the proof of Proposition 2) which describes the departure from the pest-free solution. This gives the equivalent variational system

$$
\left\{\begin{array}{l}
\dot{\tilde{x}}=f(x)-g(s) y=f(\tilde{x})-g(s)\left(\tilde{y}+y_{p}\right), \\
\dot{\tilde{y}}=h(s) y-m y+m y_{p}=h(s)\left(\tilde{y}+y_{p}\right)-m \tilde{y},
\end{array}\right.
$$

where $s=\frac{\alpha(x)}{\beta(y)}=\frac{a \tilde{x}}{b\left(\tilde{y}+y_{p}\right)+b_{0}}$.

Let the initial conditions be $\left(\tilde{x}_{0}, \tilde{y}_{0}\right)$ at $t=t_{0}$. Integrating $\frac{\dot{x}}{\tilde{x}}$, we get

$$
\int_{\tilde{x}_{0}}^{\tilde{x}(t)} \frac{1}{u} d u=\int_{t_{0}}^{t}\left(\frac{f(\tilde{x})}{\tilde{x}}-\frac{g(s)}{\tilde{x}}\left(\tilde{y}+y_{p}\right)\right) d \tau .
$$

To show that $\lim _{t \rightarrow+\infty} \tilde{x}=0$, we demonstrate the divergence of $\int_{\tilde{x}_{0}}^{\tilde{x}(t)} \frac{1}{u} d u$ to $-\infty$ as $t \rightarrow+\infty$. For $t$ large enough, we have

$$
\begin{aligned}
\int_{\tilde{x}_{0}}^{\tilde{x}(t)} \frac{1}{u} d u= & \int_{t_{0}}^{\left(\left\lfloor\frac{t_{f}}{T}\right\rfloor+1\right) T}\left(\frac{f(\tilde{x})}{\tilde{x}}-\frac{g(s)}{\tilde{x}}\left(\tilde{y}+y_{p}\right)\right) d \tau+\int_{\left(\left\lfloor\frac{t_{f}}{T}\right\rfloor+1\right) T}^{\left\lfloor\frac{t}{T}\right\rfloor T}\left(\frac{f(\tilde{x})}{\tilde{x}}-\frac{g(s)}{\tilde{x}}\left(\tilde{y}+y_{p}\right)\right) d \tau \\
& +\int_{\left\lfloor\frac{t}{T}\right\rfloor T}^{t}\left(\frac{f(\tilde{x})}{\tilde{x}}-\frac{g(s)}{\tilde{x}}\left(\tilde{y}+y_{p}\right)\right) d \tau,
\end{aligned}
$$

with $t_{f}$ defined as in Proposition 2. The first and third terms in (9) are bounded for all $t$. The second integral, however, may not necessarily be so. At this point it is useful to note that from Proposition 3, we may write

$$
\frac{g(s)}{\tilde{x}}\left(\tilde{y}+y_{p}\right) \geq \frac{\sigma(s, q)}{\tilde{x}}\left(\tilde{y}+y_{p}\right)=\frac{q g^{\prime}(0) a\left(\tilde{y}+y_{p}\right)}{q b\left(\tilde{y}+y_{p}\right)+q b_{0}+a \tilde{x}},
$$

which is an increasing function of $\left(\tilde{y}+y_{p}\right)$ and a decreasing one of $\tilde{x}$.

Because of the positivity of the system, $y$ can be lower bounded. To begin with, $y_{p}(t)+\tilde{y}(t) \geq 0$. Since from (4) $\dot{\tilde{y}} \geq-m \tilde{y}$, if $\tilde{y} \geq 0$ at some time, it remains so for the remaining time. Furthermore $\tilde{y}(t) \geq-y_{p}(t) \geq-y^{*}$ which implies that $\forall t \geq t_{0}$

$$
\tilde{y} \geq-y^{*} e^{-m\left(t-t_{0}\right)}
$$

Thus,

$$
y_{p}(t)+\tilde{y}(t) \geq y^{*}\left(e^{-m(t \quad \bmod T)}-e^{-m\left(t-t_{0}\right)}\right) .
$$

From the proof of Proposition 2, we also note that $\tilde{x} \in[0, \bar{x}]$, as $t$ is sufficiently large.

Therefore, from these two bounds, we can write

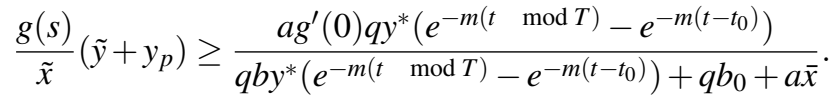

So, from (10), we can upper bound the second term of the inequality (9) as

$$
\int_{\left(\left\lfloor\frac{t}{T}\right\rfloor+1\right) T}^{\left\lfloor\frac{\left.t_{f}\right\rfloor T}{\tilde{x}}\right.}\left(\frac{f(\tilde{x})}{\tilde{x}}-\frac{g(s)}{\tilde{x}}\left(\tilde{y}+y_{p}\right)(\tau)\right) d \tau \leq \sum_{n=\left\lfloor\frac{t_{f}}{T}\right\rfloor+1}^{\left\lfloor\frac{t}{T}\right\rfloor-1} I(n),
$$


where

$$
\begin{aligned}
I(n) & =\int_{n T}^{(n+1) T}\left(\sup _{\tilde{x} \geq 0} \frac{f(\tilde{x})}{\tilde{x}}-\frac{a g^{\prime}(0) q y^{*} e^{-m(\tau-n T)}\left(1-e^{-m\left(n T-t_{0}\right)}\right)}{q b y^{*} e^{-m(\tau-n T)}\left(1-e^{-m\left(n T-t_{0}\right)}\right)+q b_{0}+a \bar{x}}\right) d \tau, \\
& =S T+\frac{a g^{\prime}(0)}{b m} \ln \left(\frac{q b y^{*} e^{-m T}\left(1-e^{-m\left(n T-t_{0}\right)}\right)+q b_{0}+a \bar{x}}{q b y^{*}\left(1-e^{-m\left(n T-t_{0}\right)}\right)+q b_{0}+a \bar{x}}\right) .
\end{aligned}
$$

$I(n)$ being decreasing in $n$, if $\lim _{n \rightarrow \infty} I(n)<0$,

$$
\lim _{t \rightarrow \infty} \sum_{n=\left\lfloor\frac{t_{f}}{T}\right\rfloor+1}^{\left\lfloor\frac{t}{T}\right\rfloor-1} I(n)=-\infty
$$

which is what we require for stability. Thus a sufficient condition for $\lim _{t \rightarrow \infty} \tilde{x}(t)=0$ is that

$$
S T+\frac{a g^{\prime}(0)}{b m} \lim _{n \rightarrow \infty} \ln \left(\frac{q b y^{*} e^{-m T}\left(1-e^{-m\left(n T-t_{0}\right)}\right)+q b_{0}+a \bar{x}}{q b y^{*}\left(1-e^{-m\left(n T-t_{0}\right)}\right)+q b_{0}+a \bar{x}}\right)<0 .
$$

Then, evaluating the limit and rearranging the above inequality yields

$$
\left(e^{-m \frac{b S}{a g^{\prime}(0)} T}-e^{-m T}\right) q b y^{*}>\left(q b_{0}+a \bar{x}\right)\left(1-e^{-m \frac{b S}{a g^{\prime}(0)} T}\right) .
$$

As previously for inequality (7), we need that

$$
\left(e^{-m \frac{b S}{a g^{\prime}(0)} T}-e^{-m T}\right)>0,
$$

which gives the first global condition (5c). Finally, recalling (3b) and rearranging (11) so that $\mu$ is the subject of formula yields

$$
\mu>\frac{q b_{0}+a \bar{x}}{q b}\left(\frac{1-e^{-m \frac{b S}{a g^{\prime}(0)} T}}{e^{-m \frac{b S}{a g^{\prime}(0)} T}-e^{-m T}}\right)\left(\frac{1-e^{-m T}}{T}\right),
$$

which is condition (5d); proving the convergence of $\tilde{y}$ to zero as well is straightforward and similar to [31]. We have then shown that $(5 \mathrm{c})$ and $(5 \mathrm{~d})$ yield global convergence of the solutions to the zero-pest solution. In order to obtain GAS, we are then left with showing that satisfying (5c) and (5d) implies that the local stability conditions (5a) and (5b) are also satisfied.

We notice that the conditions (5a)-(5b) and (5c)-(5d) have a similar form. First, since $f^{\prime}(0) \leq \sup _{x \geq 0}(f(x) / x)=S$, we directly have that $(5 \mathrm{c})$ imposes $(5 \mathrm{a})$. Next, we introduce the following notation:

$$
\underline{\mu}(Q, \lambda, T)=Q\left(\frac{1-e^{-\lambda m T}}{e^{-\lambda m T}-e^{-m T}}\right)\left(\frac{1-e^{-m T}}{T}\right) .
$$

So the LAS condition (5b) and condition (5d) can be rewritten as

$$
\mu>\underline{\mu}\left(\frac{b_{0}}{b}, \frac{b f^{\prime}(0)}{a g^{\prime}(0)}, T\right),
$$

and

$$
\mu>\underline{\mu}\left(\frac{q b_{0}+a \bar{x}}{q b}, \frac{b S}{a g^{\prime}(0)}, T\right)
$$


respectively. It is straightforward to see that $\underline{\mu}(Q, \lambda, T)$ is increasing in $Q$ and $\lambda$ and that $\frac{q b_{0}+a \bar{x}}{q b}>\frac{b_{0}}{b}$ and $\frac{b S}{a g^{\prime}(0)}>$ $\frac{b f^{\prime}(0)}{a g^{\prime}(0)}$, so that the lower bound in $(5 b)$ is smaller than in $(5 d)$. This concludes the proof of global stability.

Note that the proof on affine $\alpha$ and $\beta$ yields two types of conditions for the stability of the pest-free solution: one, condition (5a), is biological and the other, condition (5b), is managerial. These results serve as a basis for the analysis when $\alpha$ and $\beta$ are nonlinear, which is presented in the next section.

\subsection{Nonlinear parameterisation}

When the $\alpha$ and $\beta$ parameter functions are nonlinear, we suppose they are bounded within sectors given by (2). The calculations to obtain the local and global stability conditions are outlined below before summarising the results in Theorem 2.

As previously, the non-trivial local stability condition is obtained as (6), that is

$$
\int_{0}^{T}\left(\frac{\alpha^{\prime}(0) y_{p}(\tau)}{\beta\left(y_{p}(\tau)\right)}\right) d \tau>\frac{f^{\prime}(0)}{g^{\prime}(0)} T
$$

However, due to the sector conditions, the left-hand side of the inequality can only be bounded as

$$
\int_{0}^{T}\left(\frac{a_{1} y_{p}(\tau)}{b_{2} y_{p}(\tau)+b_{0}}\right) d \tau \leq \int_{0}^{T}\left(\frac{\alpha^{\prime}(0) y_{p}(\tau)}{\beta\left(y_{p}(\tau)\right)}\right) d \tau \leq \int_{0}^{T}\left(\frac{a_{2} y_{p}(\tau)}{b_{1} y_{p}(\tau)+b_{0}}\right) d \tau
$$

Therefore, for absolute stability (that is such that the stability holds for any possible $\alpha($.$) or \beta($.$) function verifying$ the sector conditions (2) [39]), it is clear that considering the lower bound in (15) will yield a sufficient condition for local stabiliy; that is, only the lower bound of $\alpha$ and the upper bound of $\beta$ are of interest. This gives

$$
\int_{0}^{T}\left(\frac{a_{1} y_{p}(\tau)}{b_{2} y_{p}(\tau)+b_{0}}\right) d \tau>\frac{f^{\prime}(0)}{g^{\prime}(0)} T .
$$

The analysis for the local stability of (3a) follows as in the previous subsection except that $a$ and $b$ are now replaced by $a_{1}$ and $b_{2}$.

The procedure to find the global stability condition is also similar to the linear case. The key point to note is that the $\sigma$-function from Proposition 3 is bounded as

$$
\sigma(s, q) \geq g^{\prime}(0) \frac{q a_{1} x}{q b_{2} y+q b_{0}+a_{1} x},
$$

provided $q$ is chosen appropriately to satisfy Proposition 3, with $K=\frac{2 a_{2} \bar{x}}{2 b_{0}+b_{1} y^{*} e^{-m T}}$. The lower bound on $\sigma$ as given in (17) is sure to lower bound $g(s)$. The analysis for global stability therefore follows as in the linear section, except that $a_{1}$ and $b_{2}$ are substituted in for $a$ and $b$ respectively. As for the local stability, due to the sector conditions (2), we thus obtain only sufficient conditions.

Theorem 2 summarises the conditions for the nonlinear parameterised system.

Theorem 2. The solution $\left(0, y_{p}(t)\right)$ of $(1)$ is LAS if

$$
f^{\prime}(0)<\frac{a_{1} g^{\prime}(0)}{b_{2}}
$$


and

$$
\mu>\underline{\mu}\left(\frac{b_{0}}{b_{2}}, \frac{b_{2} f^{\prime}(0)}{a_{1} g^{\prime}(0)}, T\right) .
$$

Furthermore, with $q$ chosen as in Proposition 3 with $K=\frac{2 a_{2} \bar{x}}{2 b_{0}+b_{1} y^{*} e^{-m T}}$, the solution is GAS if

$$
S<\frac{a_{1} g^{\prime}(0)}{b_{2}},
$$

and

$$
\mu>\underline{\mu}\left(\frac{q b_{0}+a_{1} \bar{x}}{q b_{2}}, \frac{b_{2} S}{a_{1} g^{\prime}(0)}, T\right) .
$$

We note that the minimal rates stated above are only sufficient conditions. Due to the sector conditions, a given $\mu$ value below the minimal release rate at a given $T$ value does not necessarily result in an unstable pest-free solution. The instability condition is in fact given by the lower bound of the $\alpha$ function and the higher one of the $\beta$ function as follows.

Proposition 4. The solution $\left(0, y_{p}(t)\right)$ of system (1) is unstable if

$$
f^{\prime}(0) \geq \frac{a_{2} g^{\prime}(0)}{b_{1}},
$$

or if

$$
\mu<\underline{\mu}\left(\frac{b_{0}}{b_{1}}, \frac{b_{1} f^{\prime}(0)}{a_{2} g^{\prime}(0)}, T\right) .
$$

Proof: The conditions for local instability obtained from [22] are $e^{-m \int_{0}^{T} d \tau}>1$ or $e^{\int_{0}^{T}\left(f^{\prime}(0)-\frac{g^{\prime}(0) \alpha^{\prime}(0) y_{p}(\tau)}{\beta\left(y_{p}(\tau)\right)}\right) d \tau}>1$. The former is physically impossible. After some computations, the latter however can be written as

$$
\int_{0}^{T}\left(\frac{\alpha^{\prime}(0) y_{p}(\tau)}{\beta\left(y_{p}(\tau)\right)}\right) d \tau<\frac{f^{\prime}(0)}{g^{\prime}(0)} T .
$$

Recalling (15), this instability condition is necessarily satisfied if

$$
\begin{gathered}
\int_{0}^{T}\left(\frac{a_{2} y_{p}(\tau)}{b_{1} y_{p}(\tau)+b_{0}}\right) d \tau<\frac{f^{\prime}(0)}{g^{\prime}(0)} T, \\
\Leftrightarrow b_{0}\left(e^{-m \frac{b_{1} f^{\prime}(0)}{a_{2} g^{\prime}(0)} T}-e^{-m T}\right) b_{1} y^{*}<\left(1-e^{-m \frac{b_{1} f^{\prime}(0)}{a_{2} g^{\prime}(0)} T}\right) .
\end{gathered}
$$

We note at this point that if (19a) holds, the left-hand side of inequality (21) is non-positive while the right-hand side is non-negative, so that (21) is satisfied. Otherwise, we obtain, on rearranging the inequality (21),

$$
\mu<\frac{b_{0}}{b_{1}}\left(\frac{1-e^{-m \frac{b_{1} f^{\prime}(0)}{a_{2} g^{\prime}(0)} T}}{e^{-m \frac{b_{1} f^{\prime}(0)}{a_{2} g^{\prime}(0)}}-e^{-m T}}\right)\left(\frac{1-e^{-m T}}{T}\right),
$$

which is condition (19b). 


\subsection{Interpretation of the stability results}

Our calculations have shown that a minimal release rate for pest eradication exists only if the intrinsic per capita pest growth rate $f^{\prime}(0)$ is below a threshold given by the ratio of the slope of the per capita predator consumption rate at zero pest $a g^{\prime}(0)$ to the intraspecific interference term $b$. This is a biological condition which informs the choice of the predator species. When expressed in terms of $b$, the local condition means that if the predators interfere too much with their conspecifics, no control will work even if the invasion is negligible. Similarly, a too small slope of the per capita consumption rate has identical consequences. This is intrinsically related to the prey dynamics: for an arbitrary temporal evolution of the predator population $y(t)$ (not necessarily related to model (1)), the local linearisation of the $x$ dynamics around 0 yields

$$
\dot{x}=f^{\prime}(0) x-g^{\prime}(0) \frac{a y(t)}{b_{0}+b y(t)} x,
$$

so that, the predation being upper bounded by $\frac{a g^{\prime}(0)}{b} x$, if the biological condition is not satisfied, prey-growth will always dominate predation for $x$ small; no matter how large is the predator density, pests cannot be eradicated by their natural enemies.

The dependence of the minimal release rate $\underline{\mu}$ for pest eradication with respect to the slope of the per capita consumption rate $a g^{\prime}(0)$ is also found to be monotonic decreasing. Hence a higher consumption rate of the predators requires a lower release rate to ensure that the pests are eliminated.

Analysing the influence of the interference strength requires a few more computations. We note, when the parameterisation of $\alpha(x)$ and $\beta(y)$ is affine, that the integral on the left-hand of the inequality (6) is decreasing in the interference strength $b$. When $b$ is high, $y^{*}$ needs to be increased in order to compensate for the depression in the integral value due to $b$ and hence ensure that conditions for the stability of the pest-free solution are satisfied. Since $y^{*}$ is directly proportional to $\mu$, we can infer that a higher interference strength requires a higher minimal rate for pest eradication. A similar analysis ensues on the global stability conditions and the nonlinear parameterisation case, yielding the same observation.

When the parameterisation of $\alpha$ and $\beta$ is affine, the definition of the minimal rate, that is the local stability condition on $\mu$, is a necessary and sufficient condition. In the nonlinear case, however, since we only have sufficient conditions for stability and instability, when

$$
\underline{\mu}\left(\frac{b_{0}}{b_{1}}, \frac{b_{1} f^{\prime}(0)}{a_{2} g^{\prime}(0)}, T\right)<\mu<\underline{\mu}\left(\frac{b_{0}}{b_{2}}, \frac{b_{2} f^{\prime}(0)}{a_{1} g^{\prime}(0)}, T\right),
$$

no conclusion can be drawn for the local stability of the pest-free solution.

We also find that the minimal rate is a function of the release period $T$. A more detailed analysis of this dependence is presented in section 5 . 


\section{Beyond sector conditions}

The model that we proposed with sector conditons is a wide generalization of interference models that can encompass classical models such as Beddington-DeAngelis and ratio-dependent models. The former is the paradigm of the models that can be used for the application of Theorem 1; the proofs go through an upper and lower bounding of our models by Beddington-DeAngelis models. All the developments and discussions of this paper are then directly valid for the Beddington-DeAngelis model, so that we will not elaborate beyond stating that the generality in the present paper ensures that the stability result is not an artefact related to this specific form of the model.

Therefore, we will rather analyse the conditions in the ratio-dependent limit case where $b_{0}=0$. Moreover, as interference between predator individuals may also be stronger or weaker than in the generalisation of the BeddingtonDeAngelis model we considered [20], we indicate how the stability of the pest-free solution can evolve when no sector condition can be built for the chosen interference function $\beta(y)$ i.e. when $\beta(y)$ is below or above any affine function.

\subsection{Ratio dependent model}

We have already seen that ratio-dependent models are encompassed by our analysis, by choosing $b_{0}=0$. However, the stability conditions become somewhat different due to simplifications. Indeed, the biological condition (5a) is unchanged, but the local managerial condition (5b) becomes

$$
\mu>0 .
$$

Whatever the positive release rate, if the biological condition is satisfied, the zero-pest solution becomes locally stable. This is consistent with the stability of the origin of the unforced ratio-dependent system [21], which states that, if the equivalent of our biological condition is satisfied, and if the predators cannot go extinct while preys are present, then the origin (pest-free solution, when there are no releases) is GAS; similarily, in our case, when $\mu>0$ and the biological condition is satisfied, the predators are maintained through the releaseses, so that the preys have to disappear. This stability condition $\mu>0$ is not conserved for the global stability condition (5d); we have not investigated global stability of the ratio-dependent case any further, but this major difference with the local case can be either due to an intrinsic change between the global and local stability, or to the fact that our sufficient global stability condition is too conservative.

\subsection{Weaker interference}

In this section, we will consider the case where the $\beta$ function cannot be lower-bounded by an increasing linear function; in order to do that, we will take a $\beta(y)$ function which is upper-bounded by some function $b_{0}+b y^{z}$ with $0<z<1$, whose only linear lower bound we can build is $b_{0}$ (which may or may not be valid for $\beta(y)$ ). The necessary and sufficient condition (6) for local stability here becomes, through the use of (3a)-(3b),

$$
\int_{0}^{T}\left(\frac{\alpha^{\prime}(0) \frac{\mu T e^{-m t}}{1-e^{-m T}}}{\beta\left(\frac{\mu T e^{-m t}}{1-e^{-m T}}\right)}\right) d t>\frac{f^{\prime}(0)}{g^{\prime}(0)} T .
$$


For this inequality to be satisfied, it suffices to upper bound the denominator of the left-hand-side; it becomes

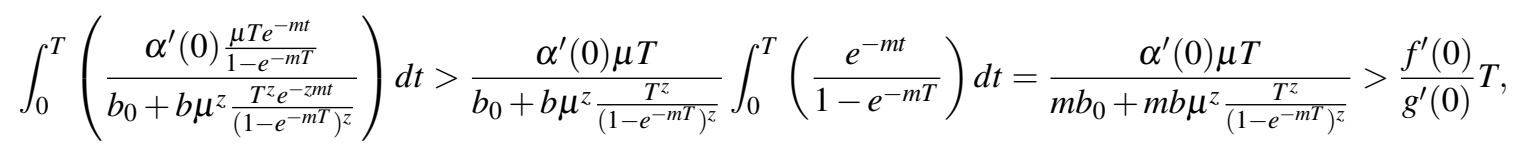

which yields the sufficient condition for stability

$$
\frac{\alpha^{\prime}(0) \mu}{m b_{0}+m b \mu^{z} \frac{T^{z}}{\left(1-e^{-m T}\right)^{z}}}>\frac{f^{\prime}(0)}{g^{\prime}(0)}
$$

Since $z \in(0,1)$, the LHS of this inequality is increasing in $\mu$ and goes to infinity as $\mu$ goes to infinity. It is then straightforward that, for a given $T$, one can always choose $\mu$ large enough so that this condition becomes satisfied. From this, we conclude that, in that case, no biological conditions like (5a) needs to be satisfied in order for the zeropest solution to be stable. The interference is weaker than the density effect on the predation: the more predators are present, the more efficient the total predation is.

\subsection{Stronger interference}

We will now see what can happen when no linear upper-bound can be found. For that, we will now consider that the interference function is lower-bounded by $b_{0}+b y^{z}$, with $z>1$. We analyse the local instability condition (20):

$$
\int_{0}^{T}\left(\frac{\alpha^{\prime}(0) \frac{\mu T e^{-m t}}{1-e^{-m T}}}{\beta\left(\frac{\mu T e^{-m t}}{1-e^{-m T}}\right)}\right) d t<\frac{f^{\prime}(0)}{g^{\prime}(0)} T .
$$

We lower-bound the denominator of the left-hand-side and have

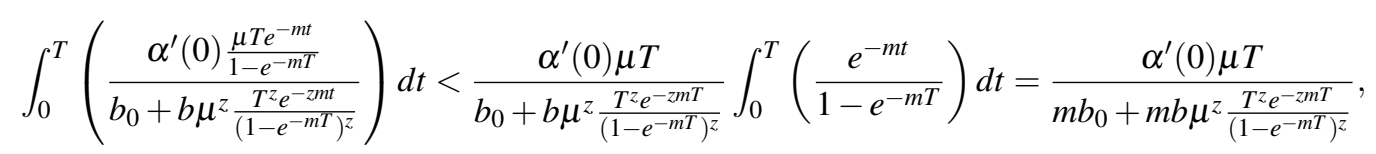

which yields the sufficient condition for instability

$$
\frac{\alpha^{\prime}(0) \mu}{m b_{0}+m b \mu^{z} \frac{T^{z} e^{-z m T}}{\left(1-e^{-m T}\right)^{z}}}<\frac{f^{\prime}(0)}{g^{\prime}(0)} .
$$

Since $z>1$, the LHS of this inequality is non-monotonic in $\mu$. More precisely it is first increasing in $\mu$ from 0 up to some value at which it starts decreasing and tends to 0 as $\mu$ tends to infinity. From this property, we conclude that, independently of the value of the parameters, the zero-pest solution is unstable (i.e. the previous condition holds true) for $\mu$ small and for $\mu$ large. This contrasts with our result in the presence of sector conditions where, when a biological condition is satisfied, increasing the release rate ensures that the zero-pest solution is stable. Efficient control is then potentially only achieved for intermediate values of the release-rate. Here, the interference is stronger than the density effect: the larger the predator-density, the less efficient the total predation is. 


\subsection{Positioning of our model}

In this section, we have shown that the sector conditions that we have presented generate a somewhat maximal family of models that, as in the Beddington-DeAngelis case, yield stability conditions consisting of a biological condition and a managerial condition stating that the release-rate must be taken large enough; this result is not related to the form of the trophic responses. Also, having identified in the previous subsections the structural changes that occur to the stability of the pest-free solution when departing from the sector conditions, we have shown the importance of considering different generic forms of interference to assess the influence they have on the efficiency of biological control.

\section{The release strategy}

Coming back to our original model, the release strategy of the pest management program can be optimised in two aspects, namely the minimal release rate for pest eradication, which we seek to minimise, and the speed of convergence to the zero-pest solution upon a pest invasion, which we would like to maximise. We investigate the effect of the release period $T$ on these two characteristic features below, in each case when the parameterisation is affine, then when it is nonlinear.

\subsection{The minimal predator release rate}

The expression for the minimal release rate required for the eradication of pests is obtained from the stability analysis presented previously.

\subsubsection{Affine parameterisation}

When the arguments of the trophic responses are considered to be affine as in subsection 3.1, the calculation of $\frac{\partial \mu}{\partial T}$ yields the following observation:

Theorem 3. For $0<\lambda<1, \underline{\mu}(Q, \lambda, T)$ is increasing in $T$ for $T>0$ and $\lim _{T \rightarrow \infty} \underline{\mu}(Q, \lambda, T)=+\infty$.

The proof, which is a bit technical, has been postponed to Appendix A.

This result applied to (5b) and (5d) has two possible interpretations. First, more frequent releases will require a smaller minimal rate to succeed in driving the pest population to zero. Second, a given rate $\mu^{*}$ will work up to a threshold release period $T^{*}$, which is defined such that

$$
\underline{\mu}\left(\frac{b_{0}}{b}, \frac{b f^{\prime}(0)}{a g^{\prime}(0)}, T^{*}\right)=\mu^{*} .
$$

Beyond this period, as the zero-pest solution is unstable, pest invasion occurs. 


\subsubsection{Nonlinear parameterisation}

When the parameterisation of $\alpha(x)$ and $\beta(y)$ is nonlinear, only general trends in the behaviour of the release rate with respect to the release period can be identified.

Thus while the precise value of the minimal release rate, which we shall denote by $\tilde{\mu}(T)$, is unknown, we know however that it is bounded as in (22). Each of the bounds have the same form as for the affine case and are each increasing in $T$. The actual $\tilde{\mu}(T)$ will therefore generally tend to increase with respect to the release period $T$ within a tube defined by these two bounds. As in the affine case, increasing the release period for a given release rate $\mu^{*}$ will at some point destabilise the pest-free solution. Due to the sector formulation, we are able to calculate that the rate $\mu^{*}$ will work up to a release period $\underline{T^{*}}$, but not beyond a $\overline{T^{*}}>\underline{T^{*}}$, defined respectively such that

$$
\underline{\mu}\left(\frac{b_{0}}{b_{2}}, \frac{b_{2} f^{\prime}(0)}{a_{1} g^{\prime}(0)}, \underline{T^{*}}\right)=\mu^{*}, \text { and } \underline{\mu}\left(\frac{b_{0}}{b_{1}}, \frac{b_{1} f^{\prime}(0)}{a_{2} g^{\prime}(0)}, \overline{T^{*}}\right)=\mu^{*} .
$$

A graphical representation of this result is presented in Figure 2. Here we have considered $\alpha(x)$ to be affine and $\beta(y)$ a Lipschitz square-like wave similar to the one given in Figure 1. The shaded tube is limited by the bounds resulting from the sector formulation and contains the actual $\tilde{\mu}(T)$ curve for the pair of $\alpha(x)$ and $\beta(y)$ functions considered. Notice that in this example the actual $\tilde{\mu}(T)$ coincides with $\underline{\mu}\left(\frac{b_{0}}{b_{2}}, \frac{b_{2} f^{\prime}(0)}{a_{1} g^{\prime}(0)}, T\right)$ for small $T$ and hedges close to $\underline{\mu}\left(\frac{b_{0}}{b_{1}}, \frac{b_{1} f^{\prime}(0)}{a_{2} g^{\prime}(0)}, T\right)$ for intermediate $T$ values. This somewhat validates the quality of the bounds obtained on $\tilde{\mu}(T)$ from the chosen sectors.

\subsection{The pest evolution rate}

When designing the biological control program, we also aim for the pest eradication to be fast enough. We may define a mathematical expression for the rate of evolution around the pest-free state as follows. At first order in $\left(x, y-y_{p}\right)$, the $x$-dynamics are

$$
\dot{x}=\left(f^{\prime}(0)-g^{\prime}(0) \frac{\alpha^{\prime}(0)}{\beta\left(y_{p}(t)\right)}\right) x,
$$

so that $\forall t$,

$$
x(t+k T)=x(t) e^{\phi(T) k T}
$$

where the exponent

$$
\phi(T)=\frac{1}{T} \int_{0}^{T}\left(f^{\prime}(0)-g^{\prime}(0) \frac{\alpha^{\prime}(0)}{\beta\left(y_{p}(\tau)\right)} y_{p}(\tau)\right) d \tau,
$$

is the mean rate of evolution of $x$ around the pest-free solution $\left(0, y_{p}\right)$. We note that $\phi<0$ when the solution is locally stable, so that $x$ converges to zero. For all positive $\phi, x$ diverges from zero. The mean speed of evolution of $x$ around $\left(0, y_{p}\right)$ depends on the magnitude of $\phi$ : the larger its magnitude, the higher the speed of evolution (divergence or convergence). This point is important in the interpretation of the subsequent results.

When $\beta\left(y_{p}\right)$ is affine, $\frac{y_{p}}{\beta\left(y_{p}\right)}$ increases with $y_{p}$ and therefore increases with $\mu$. When the parameterisation is nonlinear, $\frac{y_{p}}{\beta\left(y_{p}\right)}$ will also follow a generally increasing trend because of the sector conditions. Thus the rate $\phi$ decreases the higher the predator release rate used. When the pest-free solution is stable, this means that a higher predator 


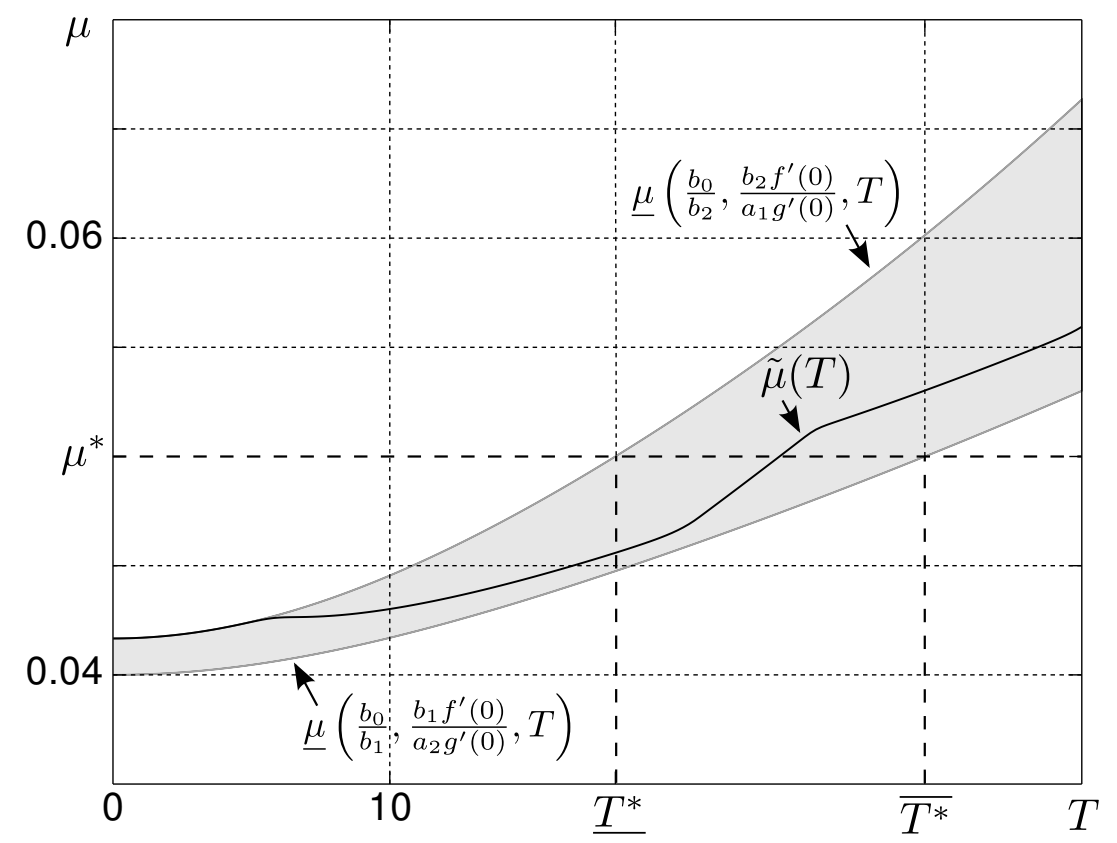

Figure 2: Minimal release rate $\tilde{\mu}(T)$ numerically computed from an affine $\alpha(x)$ and a Lipschitz square-like $\beta(y)$ shown on Figure 1. The shaded area is the tube defined by (22) to which $\tilde{\mu}(T)$ belongs. The threshold $\underline{T^{*}}$ (resp. $\overline{T^{*}}$ ) under which (resp. over) the pest-free solution is guaranteed to be locally stable (resp. unstable) is also shown for $\mu^{*}=0.05$. 
release rate implemented ensures a faster pest eradication (since $\phi$ becomes more negative). In practice however, due to financial constraints, this rate has a maximal fixed value, which in turn limits the speed of pest elimination, at a given release period.

However, we note that this evolution rate $\phi$ is also dependent on the release period, related to the release program. Here, for a given release rate $\mu$, the question is how to deploy the predators in time in order to maximise the speed of eradication.

\subsubsection{Affine parameterisation}

When the arguments of the trophic responses are considered to be affine as in subsection 3.1, the convergence rate $\phi(T)$ is calculated as

$$
\phi(T)=f^{\prime}(0)+\frac{g^{\prime}(0) a}{b m T} \ln \left(\frac{b y^{*} e^{-m T}+b_{0}}{b y^{*}+b_{0}}\right) .
$$

Notice directly that, in the ratio-dependent case $\left(b_{0}=0\right)$, this rate becomes $f^{\prime}(0)-\frac{g^{\prime}(0) a}{b}$, which is independent of the release-period $T$. Otherwise, the following theorem then applies.

Theorem 4. Suppose that local stability conditions are satisfied. Then for a given $\mu$, the average rate of evolution of $x$ to the pest-free solution is an increasing function of the release period.

Proof: Recalling (3b), for a given $\mu$, we can write

$$
\phi(T)=f^{\prime}(0)+\frac{g^{\prime}(0) a}{b m T} \ln \left(\frac{b \mu T e^{-m T}+b_{0}\left(1-e^{-m T}\right)}{b \mu T+b_{0}\left(1-e^{-m T}\right)}\right) .
$$

This equation can be rearranged with $\mu$ as the subject of the formula and expressed using the $\underline{\mu}$-function as follows:

$$
\mu=\frac{b_{0}}{b}\left(\frac{1-e^{-\frac{b}{a}\left(\frac{f^{\prime}(0)-\phi(T)}{g^{\prime}(0)}\right) m T}}{e^{-\frac{b}{a}\left(\frac{f^{\prime}(0)-\phi(T)}{g^{\prime}(0)}\right) m T}-e^{-m T}}\right) \frac{1-e^{-m T}}{T}=\underline{\mu}\left(\frac{b_{0}}{b}, \frac{b}{a}\left(\frac{f^{\prime}(0)-\phi(T)}{g^{\prime}(0)}\right), T\right) .
$$

To determine the sign of $\frac{\partial \phi}{\partial T}$, we make use of the chain rule:

$$
\frac{\partial \mu}{\partial T}=\frac{\partial \underline{\mu}}{\partial T}+\frac{\partial \underline{\mu}}{\partial \phi} \frac{\partial \phi}{\partial T}
$$

For a given $\mu, \frac{\partial \mu}{\partial T}=0$. We note that independently of the sign of $\phi(T)$, from its definition in (24) and since $b y^{*}>0$, for $T>0$

$$
\frac{b}{a}\left(\frac{f^{\prime}(0)-\phi(T)}{g^{\prime}(0)}\right)=-\frac{1}{m T} \ln \left(\frac{b y^{*} e^{-m T}+b_{0}}{b y^{*}+b_{0}}\right)<-\frac{1}{m T} \ln \left(e^{-m T}\right)=1 .
$$

Furthermore, since $0<\frac{b y^{*} e^{-m T}+b_{0}}{b y^{*}+b_{0}}<1$, we can deduce that

$$
-\frac{1}{m T} \ln \left(\frac{b y^{*} e^{-m T}+b_{0}}{b y^{*}+b_{0}}\right)>0 .
$$


Therefore we can establish that

$$
0<\frac{b}{a}\left(\frac{f^{\prime}(0)-\phi(T)}{g^{\prime}(0)}\right)<1
$$

So, we can apply Theorem 3 and deduce that $\frac{\partial \mu}{\partial T}>0$. Furthermore

$$
\frac{\partial \underline{\mu}}{\partial \phi}=-\frac{m b_{0}}{a g^{\prime}(0)} \frac{\left(1-e^{-m T}\right)^{2} e^{-\frac{b}{a}\left(\frac{f^{\prime}(0)-\phi(T)}{g^{\prime}(0)}\right) m T}}{\left(e^{-\frac{b}{a}\left(\frac{f^{\prime}(0)-\phi(T)}{g^{\prime}(0)}\right) m T}-e^{-m T}\right)^{2}}<0 .
$$

So for (25) to be satisfied, $\frac{\partial \phi}{\partial T}>0$.

When the zero-pest solution is stable, $\phi(T)$ is negative and the pest population asymptotically tends towards zero. The above result therefore means that the rate of evolution towards zero becomes less negative, that is its magnitude decreases, as the period of release is increased. The speed of convergence towards the pest-free solution decreases until zero.

Figure 3 illustrates this result. The convergence of $x$ to zero for three different release periods is shown. In all three cases, $\mu$ is identical and sufficiently high to ensure the stability of this solution. So $\phi$ is negative and the convergence of $x$ to zero is faster for smaller release periods.

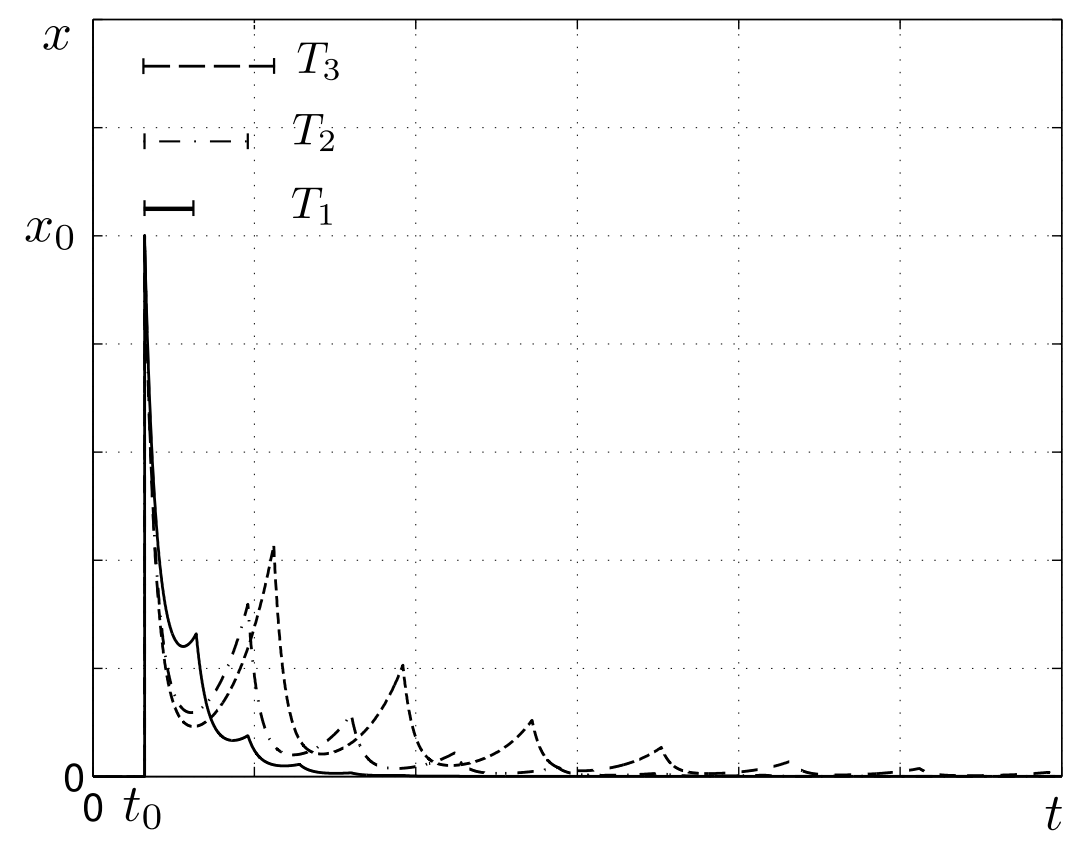

Figure 3: Pest convergence for a given $\mu$ at different release periods, $T_{1}<T_{2}<T_{3}$. As the period of release is increased, the pest population converges less quickly to zero. 
The proof of Theorem 4 actually tells us more than Theorem 4 itself. For positive $\phi(T)$, that is when the stability of the pest-free system is not guaranteed, increasing the period of releases increases the magnitude of $\phi$. In fact, it is the rate of invasion that is increased. We remark here that keeping $T$ as small as possible is favourable even if $\phi$ is positive since it would be delaying the invasion.

\subsubsection{Nonlinear parameterisation}

When the parameterisation of $\alpha(x)$ and $\beta(y)$ is nonlinear, we can determine the rate of evolution to be enclosed within a tube, the upper and lower bounds of which are related to the sector conditions. Recalling (15), we can bound $\phi$ defined in (23) as

$$
\phi\left(T ; a_{2},\left(b_{1} y_{p}+b_{0}\right)\right) \leq \phi\left(T ; \alpha^{\prime}(0), \beta\left(y_{p}\right)\right) \leq \phi\left(T ; a_{1},\left(b_{2} y_{p}+b_{0}\right)\right)
$$

From the proof of Theorem 4 , both $\phi\left(T ; a_{2},\left(b_{1} y_{p}+b_{0}\right)\right)$ and $\phi\left(T ; a_{1},\left(b_{2} y_{p}+b_{0}\right)\right)$ are increasing in $T$. From the bounds imposed in (27), we can deduce that $\phi(T)$ will follow a generally increasing trend with respect to the release period $T$. The actual speed of convergence towards the pest-free solution will tend to decrease. Similarly to the behaviour of $\mu$ with respect to $T$ described above, while the value of $T$ at the actual crossover of $\phi$ is unknown, the threshold values $\overline{T^{*}}$ and $\underline{T^{*}}$ are obtained as

$$
\phi\left(\underline{T^{*}} ; a_{1},\left(b_{2} y_{p}+b_{0}\right)\right)=0 \text { and } \phi\left(\overline{T^{*}} ; a_{2},\left(b_{1} y_{p}+b_{0}\right)\right)=0 .
$$

Figure 4 shows the variation of the rate of evolution $\phi$ with respect to the release period $T$, with the release rate fixed at $\mu^{*}=0.05$. As for the simulation to calculate $\tilde{\mu}(T)$ in the previous section, $\alpha(x)$ is affine and $\beta(y)$ Lipschitz square-like. All parameters are identical. The shaded tube is limited by the bounds obtained from the sector conditions and contains the actual $\phi$ that was solved numerically. The two thresholds on the release period $\left(\underline{T^{*}}\right.$ and $\left.\overline{T^{*}}\right)$ are equal to ones in Figure 2. Once again, for this example, we note that the actual $\phi$ coincides with the upper bound $\phi\left(\overline{T^{*}} ; a_{2},\left(b_{1} y_{p}+b_{0}\right)\right)$ for small $T$ and approaches close to $\phi\left(\underline{T^{*} ; a_{1}},\left(b_{2} y_{p}+b_{0}\right)\right)$ for intermediate $T$ values. The quality of our bounds is demonstrated.

Between $\underline{T^{*}}$ and $\overline{T^{*}}$ no conclusion can be drawn. We can simply say that the results in the the affine as well as in the nonlinear parameterisation case tend to favour the choice of a small release period.

\section{Discussion}

The work presented in this paper focuses on the existence and stability conditions of the pest-free solution of a semi-discrete predator-prey system within a biological control program. Our model accounts for intraspecific interference among the predators. The trophic responses are defined qualitatively with respect to the prey and predator population generalising the classical Beddington-DeAngelis formulation. We also provide an explicit expression for the size of predator releases taking place at regular time intervals defined as the predator release rate and the release period. Because of this formulation, we are able to obtain, from the stability analysis of the pest-free solution, two 


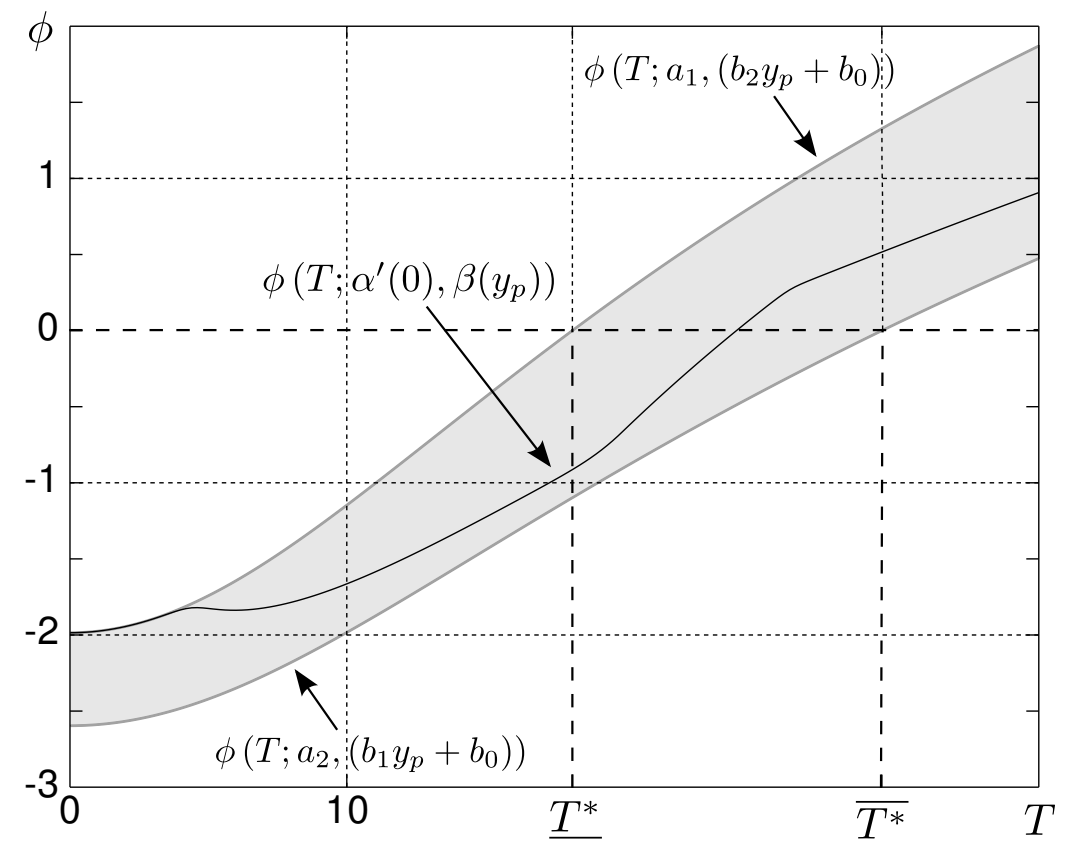

Figure 4: Mean rate of evolution $\phi(T)$ numerically computed from $\mu=0.05$, an affine $\alpha(x)$ and a Lipschitz square-like $\beta(y)$ shown on Figure 1. The shaded area is the tube defined by (27) to which $\phi$ belongs. The threshold $\underline{T^{*}}$ (resp. $\overline{T^{*}}$ ) under which (resp. over) the pest-free solution is guaranteed to be locally stable (resp. unstable) is also shown. 
types of conditions: the first one of biological nature and the second in terms of implementation of the biological control program that would inform the choice of the predator species and the strategy of its release respectively.

The biological condition determines an upper threshold on the interference parameter $b$, beyond which no control would be possible - this even if the invasion of pests were to be very small. This threshold is proportional to the slope of the per capita predator consumption rate at low pest invasions $a g^{\prime}(0)$, and is inversely proportional to the per capita pest growth rate.

In practice, this mathematical result can be interpreted thus: the ideal predator species for biological control has sufficiently low intraspecific interfering behaviour $b$ and high consumption efficiency $a g^{\prime}(0)$. Concerning the release strategy, we show that the rate of predator release $\mu$ that guarantees the stability of the pest-free solution must be larger than a function dependent in the release period $T$. When the parameterisation is affine, a given rate that is smaller than this threshold value results in the pest-free solution being unstable. Our analysis in fact yields a necessary condition for the local stability of this solution. When the parameterisation is nonlinear, there are two distinct thresholds: a lower bound on the rate for stability and an upper bound for instability of the pest-free solution. Both conditions are only sufficient. The dependence of the abovementioned threshold in $T$ is presented explicitly, and generalises the work of $[43,32]$ on explicit Beddington-DeAngelis trophic functions in that we ensure that the conclusions we draw are generic and not specifically related to a model form; additionnally, we provide global stability conditions that guarantee the efficiency of the biological control for any initial size of infestation, as opposed to local stability conditions that only ensure pest eradication in cases of small outbreaks. Our study of the release rate condition's dependence on $T$ also reveals that small frequent releases lower the minimal release rate required for pest eradication and maximise the speed of convergence to the pest-free solution. This means that in practice, small frequent releases are to be favoured over large less frequent ones.

From a modelling point of view, incorporating interference effects within the trophic response results in the dependence of the minimal control for pest eradication on the period of release. This dependence confirms the need for an impulsive model since a real-life implementation of the control effort can only be discrete in time. If we had modelled it continuously, through the incorporation of a $+\mu$ control term in the $\dot{y}$ equation instead of the discrete equation in model (1) (resulting in a continuous time model "equivalent" to model (1) obtained by taking $T \rightarrow 0$ ), we would have deduced incorrect predictions on the minimal rate $\mu$, although we would have found the same necessary biological condition. The existence of a threshold period beyond which biological control is disrupted appears to be an inherent feature of intraspecific interference effects. In fact, it is also apparent when the interference is modelled through a density dependent increase of the predator mortality rate [32] rather than by modifying the trophic function and, although we do not show it here, similar properties can be derived in the case of weak and strong interference as presented in subsections 4.2 and 4.3. Conversely, no such dependence in $T$ is obtained when no interference effects are modelled [27].

Our study also reveals that one should take care when deriving ecological systems management practices from mathematical models. The qualitative framework proposed in this paper reduces the risks of modelling artefacts 
and allows to identify properties that seem to be universal when predators interfere (e.g. the existence of a critical release period $T$ ). However, the conclusions we draw on the stability conditions of the pest-free solution appear to be strongly related to the form of "moderate interference" exemplified by the class of qualitative Beddington-DeAngelis trophic responses we proposed. Indeed, as shown in Section 4, weaker or stronger interference types, as well as ratiodependence, result in different pest-free stability conditions and hence different management advices. For instance in case of strong intrapredatory interference, it is unwise to increase too much the predator release rate in the hope it will yield better pest control: it can, by itself, disrupt pest control. On the contrary, for weak or moderate interference, a higher predator release rate facilitates pest elimination, while it has no effect in the ratio-dependent case, where pest control is achieved with the same efficiency independently of the release rate.

The last take home message of this paper is that investigating to which "maximal" class of models generic statements apply should not be disregarded: it allows to identify what biological characteristics are at their core, and, conversely, those that would break them up.

\section{References}

[1] B. K. Agarwala, H. Yasuda, and Y. Kajita. Effect of conspecific and heterospecific feces on foraging and oviposition of two predatory ladybirds: role of fecal cues in predator avoidance. Journal of Chemical Ecology, 29:357-376, 2003.

[2] R. Arditi and L.R. Ginzburg. Coupling in predator-prey dynamics: ratio-dependence. Journal of Theoretical Biology, 139:311-326, 1989.

[3] J. R. Beddington. Mutual interference between parasites or predators and its effect on searching efficiency. Journal of Animal Ecology, 44:331-340, 1975.

[4] G. Buffoni, M. P. Cassinari, M. Groppi, and M. Serluca. Modelling of predator-prey trophic interactions. Part I: two trophic levels. Journal of Mathematical Biology, 50:713-732, 2005.

[5] G. Buffoni and G. Gilioli. A lumped parameter model for acarine predator-prey population interactions. Ecological Modelling, 170(2-3):155171, December 2003.

[6] A. Byers, D. Giovannucci, and P. Liu. Value adding standards in the North American food market. trade opportunities in certified products for developing countries. Commodities and Trade Technical paper 11, FAO Trade and Markets Division, Rome, 2008.

[7] A. Damon. Evaluation of current techniques and new approaches in the use of Cephalonomia stephanoderis (Hymenoptera: Bethylidae) as a biological control agent of the coffee berry borer, Hypothenemus hampei (Coleoptera: Scolytidae), in Chiapas, Mexico. PhD thesis, University of London, 2000.

[8] A. Damon and J. Valle. Comparison of two release techniques for the use of Cephalonomia stephanoderis (Hymenoptera: Bethylidae), to control the coffee berry borer Hypothenemus hampei (Coleoptera: Scolytidae) in Soconusco, southeastern Mexico. Biological Control, 24:117-127, 2002.

[9] D. L. DeAngelis, R. A. Goldstein, and R.V. O'Neill. A model for trophic interaction. Ecology, 56:881-892, January 1975.

[10] Z. S. Duan, J. Z. Wang, G. R. Chen, and L. Huang. Stability analysis and decentralized control of a class of complex dynamical networks. Automatica, 44(4):1028-1035, April 2008.

[11] H. T. M. Eskola and S. A. H. Geritz. On the mechanistic derivation of various discrete-time population models. Bulletin Of Mathematical Biology, 69(1):329-346, January 2007.

[12] E. S. Everleigh and D. A.. Chant. Experimental studies: the effects of predator density on prey consumption, predator searching efficiency, and the functional response to prey density (Acarina: Phytoseiidae). Canadian Journal of Zoology, 60:611-629, 1982.

[13] R. W. Flowers, S. M. Salom, and L. T. Kok. Competitive interactions among two specialist predators and a generalist predator of hemlock woolly adelgid, Adelges tsugae (Hemiptera: Adelgida) in south-western Virginia. Agricultural and Forest Entomology, 8:253-262, 2006. 
[14] U. Gerson. Intraguild predation and other interactions among acarine biocontrol agents. In U. Gerson, R. L. Smiley, and R. Ochoa, editors, Mites (Acari) for pest control, pages 360-366. Blackwell Publishing Ltd, 2007.

[15] M. P. Hassell and G. C. Varley. New inductive population model for insect parasites and its bearing on biological control. Nature, 223:11331137,1969

[16] C. S. Holling. Some characteristics of simple types of predation and parasitism. Canadian Entomologist, 91:385-398, 1959.

[17] T.S. Hu, B. Huang, and Z.L. Lin. Absolute stability with a generalized sector condition. IEEE Transactions on Automatic Control, 50(11):1872-1877, 2004.

[18] A. Janssen, J. Bruin, G. Jacobs, R. Schraag, and M. W. Sabelis. Predators use volatiles to avoid prey patches with conspecifis. Journal of Animal Ecology, 66:223-232, 1997.

[19] H. Khalil. Nonlinear Systems. Prentice-Hall, Upper Saddle River, New Jersey, 3rd edition, 2002.

[20] M. Koen-Alonso. A process-oriented approach to the multispecies functional response. In N. Rooney, K.S. McCann, and D.L.G. Noakes, editors, From Energetics to Ecosystems: The Dynamics and Structure of Ecological Systems., pages 1-36. Springer, 2007.

[21] Y. Kuang and E. Beretta. Global qualitative analysis of a ratio-dependent predator-prey system. Journal of Mathematical Biology, 36(4):389406, March 1998.

[22] A. Lakmeche and O. Arino. Bifurcation of non-trivial periodic solutions of impulsive differential equations arising in chemotherapeutic treatment. Dynamics of Continuous, Discrete and Impulsive Systems, 7(2):265-287, 2000.

[23] M. R. Liberzon. Essays on the absolute stability theory. Automation and remote control, 67(10):1610-1644, October 2006.

[24] B. Liu, Y. J. Zhang, and L. S. Chen. The dynamical behaviors of a Lotka-Volterra predator-prey model concerning integrated pest management. Nonlinear Analysis - Real World Applications, 6(2):227-243, April 2005.

[25] A.I. Lur'e and V.N. Postnikov. On the theory of stability of the controlled systems. Prikl. Mat. Mekh., 8(3):246-248, 1944.

[26] L. Mailleret and F. Grognard. Optimal release policy for prophylactic biological control. In Positive Systems: Theory and Applications, volume 341 of Lecture Notes in Control and Information Sciences, pages 89-96. Springer Verlag, 2006.

[27] L. Mailleret and F. Grognard. Global stability and optimisation of a general impulsive biological control model. Mathematical Biosciences, 221-2:91-100, 2009.

[28] L. Mailleret and V. Lemesle. A note on semi-descrete modelling in life sciences. Philosophical Transactions of the Royal Society, A., 367:4779-4799, 2009.

[29] G. Nachman. The effects of prey patchiness, predator aggregation and mutual interference on the functional response of Phytoseiulus persimilis feeding on Tetranychus urticae (Acari: Phytoseiidae, Tetranychidae). Experimental and Applied Acarology, 38:87-111, 2006.

[30] K. Negi and S. Gakkhar. Dynamics in a Beddington-DeAngelis prey-predator system with impulsive harvesting. Ecological Modelling, 206:421-430, 2007.

[31] S. Nundloll, L. Mailleret, and F. Grognard. The effect of partial crop harvest on biological pest control. Rocky Mountain Journal of Mathematics, 38(5):1633-1662, 2008.

[32] S. Nundloll, L. Mailleret, and F. Grognard. Two models of interfering predators in impulsive biological control. Journal of Biological Dynamics, 4:102-114, 2010.

[33] J. P. Reganold, J. D. Glover, P. K. Andrews, and H. R. Hinman. Sustainability of three apple production systems. Nature, 410:926-930, 2001.

[34] T. Royama. Analytical Population Dynamics. Chapman and Hall, London, 1992.

[35] G. D. Ruxton and W. S. C. Gurney. Interference and generation cycles. Theoretical Population Biology, 42:235-253, 1992.

[36] B. Shulgin, L. Stone, and Z. Agur. Pulse vaccination strategy in the sir epidemic model. Bulletin of Mathematical Biology, 60:1123-1148, 1998.

[37] S. Tang, Y. Xiao, and R. A. Chen, L. Cheke. Integrated pest management models and their dynamical behaviour. Bulletin of Mathematical Biology, 67:115-135, 2005.

[38] J. Umbanhowar and S. Maron, J. Harrison. Density-dependent foraging behaviours in a parasitoid lead to density-dependent parasitism of its host. Oecologia, 137:123-130, 2003. 
[39] M. M. Vidyasagar. Nonlinear Systems Analysis. Prentice-Hall, Englewood Cliffs, New Jersey, 1993.

[40] M. E. Visser, T. H. Jones, and G. Driessen. Interference among insect parasitoids: multi-patch experiment. Journal of Animal Ecology, 68:108-120, 1999.

[41] H. Yasuda, T. Takagi, and K. Kogi. Effects of conspecific and heterospecific larval tracks on the oviposition behaviour of the predatory ladybird, Harmonia axyridis (Coleoptera: Coccinellidae). European Journal of Entomology, 97:551-553, 2000.

[42] H. Zhang, P. Georgescu, and L. S. Chen. An impulsive predator-prey system with Beddington-DeAngelis functional response and time delay. International Journal of Biomathematics, 1(1):1-17, March 2008.

[43] S. Zhang and L. S. Chen. A study of predator-prey models with the Beddington-DeAngelis functional response and impulsive effect. Chaos, Solitons and Fractals, 27:237-248, 2006.

\section{A. Proof of Theorem 3}

The function can be rewritten as

$$
\underline{\mu}(Q, \lambda, T)=Q m \frac{2 \sinh \left(\frac{\lambda m T}{2}\right) \sinh \left(\frac{m T}{2}\right)}{\sinh \left(\frac{(1-\lambda) m T}{2}\right) m T}=Q m \frac{2}{\left(\operatorname{coth}\left(\frac{\lambda m T}{2}\right)-\operatorname{coth}\left(\frac{m T}{2}\right)\right) m T} .
$$

Its derivative is calculated as

$$
\begin{aligned}
\frac{d \underline{\mu}}{d T} & =2 Q m^{2} \frac{-\left(\operatorname{coth}\left(\frac{\lambda m T}{2}\right)-\operatorname{coth}\left(\frac{m T}{2}\right)\right)+\left(\frac{\frac{\lambda m T}{2}}{\sinh ^{2}\left(\frac{\lambda m T}{2}\right)}-\frac{\frac{m T}{2}}{\sinh ^{2}\left(\frac{m T}{2}\right)}\right)}{m^{2} T^{2}\left(\operatorname{coth}\left(\frac{\lambda m T}{2}\right)-\operatorname{coth}\left(\frac{m T}{2}\right)\right)^{2}} \\
& =\frac{2 Q\left(\Gamma\left(\frac{\lambda m T}{2}\right)-\Gamma\left(\frac{m T}{2}\right)\right)}{T^{2}\left(\operatorname{coth}\left(\frac{\lambda m T}{2}\right)-\operatorname{coth}\left(\frac{m T}{2}\right)\right)^{2}}
\end{aligned}
$$

where

$$
\Gamma(u)=\frac{u}{\sinh ^{2} u}-\operatorname{coth} u
$$

The denominator is positive so the sign of $\frac{d \mu}{d \bar{T}}$ will depend on that of the numerator. We note that

$$
\frac{d \Gamma}{d u}=\frac{2}{\sinh ^{2} u}(1-u \operatorname{coth} u)
$$

is negative for all $u \geq 0 . \Gamma(u)$ is therefore a decreasing function of $u$. Since $\lambda<1$, we deduce that

$$
\Gamma\left(\frac{\lambda m T}{2}\right)>\Gamma\left(\frac{m T}{2}\right)
$$

so that

$$
\frac{d \underline{\mu}}{d T}>0 .
$$

Finally,

$$
\lim _{T \rightarrow+\infty} \underline{\mu}=\lim _{T \rightarrow+\infty} \frac{Q\left(1-e^{-m T}\right)\left(1-e^{-\lambda m T}\right)}{\left(1-e^{-(1-\lambda) m T}\right)} \frac{e^{\lambda m T}}{T}=+\infty
$$

